\title{
ENERGY EFFICIENT MAXIMUM LIFETIME ROUTING FOR WIRELESS SENSOR NETWORK
}

\author{
Sourabh Jain ${ }^{1}$, Praveen Kaushik ${ }^{1}$, Jyoti Singhai ${ }^{2}$ \\ ${ }^{1}$ Department of Computer Sciene and Engineering \\ Maulana Azad National Institute of Technology, Bhopal \\ sourabh52@gmail.com, kaushikp@manit.ac.in \\ ${ }^{2}$ Department of Electronic and Communication \\ Maulana Azad National Institute of Technology, Bhopal \\ j.singhai@gmail.com
}

\begin{abstract}
In wireless sensor network, sensors or nodes are generally battery powered devices. These nodes have limited amount of initial energy that are consumed at different rates, depending on the power level. For maximizing the lifetime of these nodes most routing algorithm in wireless sensor networks uses the energy efficient path. These energy efficient routing algorithms select a best path for data transmission and consume less energy. But a single best path puts extra load to a specific node causing lower lifetime. This paper proposes an energy efficient maximum lifetime routing algorithm. It is based on a greedy heuristic technique to maximize lifetime of the system. For achieving maximum system lifetime proposed algorithm uses the energy cost of links for constructing energy efficient path. The Simulation results demonstrate that EEMLR algorithm significantly minimizes energy consumption of each node and balanced the energy for entire network as well as extend the network lifetime.
\end{abstract}

\section{KEYWORDS}

Battery power, Energy Cost, Lifetime, Routing, Wireless Sensor Network

\section{INTRODUCTION}

Wireless sensor networks (WSN's) have attracted a great deal of research attention due to their wide-range of potential applications. Applications of WSN include battlefield surveillance, biological detection, medical monitoring, home security and inventory tracking. This type of network consists of a group of nodes and each node has limited battery power. There may be many possible routes available between two nodes over which data can flow. Assume that each node generated some information and this information needs to be delivered to a destination node. Any node in the network can easily transmit their data packet to a distance node, if it has enough battery power. If any node is far from its neighbour node then large amount of transmission energy is required to transmit the data to distance node. After every transmission, remaining energy of this node decreases and some amounts of data transmission this node will be eliminated from the network because of empty battery power and in similar situation there will be a condition that no node is available for data transmission and overall lifetime of network will decreases. Whereas network lifetime is define as the time until the first node in the network dies. For maximizing the network lifetime, data should be routed such that energy expenditure is fair 
International Journal Of Advanced Smart Sensor Network Systems ( IJASSN ), Vol 2, No.1, January 2012

among the nodes in proportion to their energy reserved, instead of routing the data to a path that minimize consumed power.

In this paper, we propose greedy heuristic based routing algorithm to maximize network lifetime in terms of first node death. Proposed approach generates an energy efficient routing path that spans all the sensor nodes. Nodes transmit some amount of data in that path and then energy efficient path is recalculated.

The organization of the rest of the paper is as follows. Section 2 discusses about related work. Section 3 describes the some assumptions. In section 4, we define energy efficient maximum lifetime routing algorithm. Next, in section 5, we describe performance evaluation. Finally in section 6 , some concluding remarks are made.

\section{RELATED WORK}

In wireless sensor network data transmission is very expensive in terms of energy consumption, while data processing consumes significantly less [1]. The energy cost of transmitting a single bit of information is approximately the same as that needed for processing a thousand operations in typical sensor node [2]. The communication subsystem has much higher energy consumption than the computation subsystem. It has been shown that transmitting one bit may consume as much as executing a few thousand instruction. On the other hand the nodes in a sensor network may not be charged once their energy drained so the lifetime of the network depends crucially on the energy of the nodes. Efficient utilization of energy is crucial to the WSNs. The sensors are extremely energy bounded, hence the network formed by these sensors are also energy constrained. The communication devices on these sensors are small and have limited power and sensing ranges. A routing protocol coordinates the activities of individual nodes in the network to achieve global goals and does it in an efficient manner. Hence lifetime of network depends on appropriate routing protocol.

Most of the earlier works on energy efficient routing in wireless sensor network uses the minimum total energy (MTE) routing for data transmission approach in this work to minimize the energy consumption to reach the destination was by sending the traffic to same path but if all the traffic follows the same path then all the nodes of that path will depleted their energy quickly [3]. Instead of trying to minimize the consumed energy the main objective is to maximize the lifetime of the system [4]. As in [4] the maximum lifetime problem is a linear programming problem and solvable in polynomial time. In this works Chang and Tassiulas proposed energy efficient routing algorithms such as flow redirection and maximum residual energy path routing. Flow redirection is the redirection based algorithm where some amount of flow is redirected from smallest longest length path to largest longest length path. Where largest longest length path is the path in which has largest capacity in terms of battery power and have less energy consumption per bit transmission. MREP algorithm augments the flow on the path whose minimum residual energy after the flow augmentation will be longest. In this work considers the single destination version of the problem.

As in [5] lifetime maximization problem is extended to multicommodity case, where each commodity has their own set of destination. Chang and Tassiulas [5] proposed flow augmentation and flow redirection algorithms for the set of origin and destination nodes and formulated the routing problem with the objective of maximizing the system lifetime. [4],[5] proposed maximizing the lifetime of a network when message rate is known but Q. Li, J. Aslam and D. Ras proposed max-min zPmin and zone based routing algorithms. These are the online, hierarchical and scalable algorithms that do not rely on knowing the message rate and optimize the lifetime of 
network. max-min zPmin algorithm combines the benefit of selecting the path with the minimum energy consumption and the path that maximize the minimal residual power of the node in the network. Scalability of this algorithm is provided in zone based routing. In zone based routing it's systematize the network structurally in geographical zones, and hierarchically to control routing across the zones [4].

Routing algorithms [4]-[6] consider energy consumption on sender side only, but in [7] the maximum lifetime routing problem is extended to include the energy consumption at the receive. Author used flow redirection algorithm as in [7] and the objective of this algorithm is to find the best link cost function which will lead to the maximization of the system lifetime and also consider the energy expenditure for unit data transmission at receiver end also.

The relation of maximizing the minimum lifetime of the nodes to minimizing the energy cost per packet was defined as in [4]-[7] but this relation take one step further to provide a delay guarantee in the time the packets reach their destination, while maximizing network lifetime [8]. Routing algorithms used as in [8] aims to give delay guarantee on the arrival of packets at the Access Point (AP) while generating energy efficient path.

C. Pandana and R. Liu proposed keep connect routing algorithm for network capacity maximization in energy constrained ad hoc network. Keep connect algorithm finds the weight of node based on how many components are connected with this node. Weight of the node can be thought as the importance of the node. Most important node is the node that results in large number of disconnected component as it dies. The proposed $\mathrm{KC}$ algorithm along with flow augmentation or with Minimum Total Energy algorithm provide the best result such as these combine algorithm provide maximum connectivity of the network as well as maximize the lifetime of network [9].

K. Kar, M Codialam, T. V. Lakshman and L. Tassiulas provided routing algorithm for network capacity maximization in energy constrained ad hoc network [10]. G. Anastasi, M. Conti, M. D. Francesco and A. passarella discussed various energy conservation schemes in wireless sensor network. To reduce power consumption in wireless sensor network, they identified three main enabling techniques, namely, duty cycling, data-driven approach and mobility [11].

Distributed energy balanced routing is proposed as in [12]. This routing algorithm uses the energy balance path for data transmission. It firstly calculates the total energy cost of all the paths from source node to base station and then select energy efficient path for data transmission. But distributed energy balanced routing algorithm considers a network scenario where few nodes can communicate with base station. For large network DEBR algorithm is not works properly, a more precise routing algorithm and problem definition is required for this class of scenario.

\section{Assumptions}

- Network is static.

- Energy consumption at the receiver and energy consumption at the unintended receiver nodes that overhear the transmission is not included.

- Consider a directed graph $\mathrm{G}(\mathrm{V}, \mathrm{A})$ where $\mathrm{V}$ is the set of all nodes and $\mathrm{A}$ is the set of all directed links $(m, n)$ where $m, n$ A.

- Let $\mathrm{P}_{\mathrm{m}}$ be the set of nodes that can be reached by node $\mathrm{m}$ with a certain power level in its dynamic range, where link $(m, n)$ exists, if $n \quad S_{m}$.

- Let each node $m$ have the initial battery energy $E_{m}$

- Let $g_{\mathrm{m}}$ be the rate at which information is generated at node $\mathrm{m}$. 
- The transmission energy required for node $\mathrm{m}$ to transmit a bit to its neighboring node $\mathrm{n}$ is $\mathrm{e}_{\mathrm{mn}}$,

- The rate at which information transmitted from node $\mathrm{m}$ to node $\mathrm{n}$ is called the flow $\mathrm{f}_{\mathrm{mn}}$

- $\quad m$ and ${ }_{n}$ are the residual energy of node $m$ and node $n$ respectively.

\section{Energy Efficient Maximum Lifetime Routing Algorithm}

The proposed routing algorithm uses shortest energy cost path that maintained the energy balance for entire network. For energy efficiency algorithm uses greedy heuristic path. For energy efficient greedy heuristic optimal path algorithm calculate the energy cost of each and every link in the network. This means it finds a subset of the links that forms an optimal path that includes every node, where total cost of all the links in that path is minimized.

The information of energy available in the nodes is used to compute greedy heuristic path, and to balance the energy consumption across all nodes. Node that has minimum battery power will drain out their battery power quickly and would be the first one to die. So node with less energy can be added later in greedy heuristic optimal path because energy cost for a transmission from this node will be the maximum.

When network is setup each node can broadcast their residual energy information. All the nodes in network know the residual energy of neighbouring nodes. Initially we assume that base station is in greedy heuristic optimal path. Algorithm can calculate greedy heuristic path using the energy cost function defined in equation (1). The node of the network added to the optimal path at each point is that node adjacent to a node of the optimal path by the link of minimum energy cost. The link of the minimum cost becomes in a path are connecting the new node to the path.

When all the nodes of the network have been added to the optimal path, a greedy heuristic route is constructed for a network. All the nodes of this greedy heuristic network can transmit their data on energy efficient path. After transmitting the ' $\theta$ ' amount of data flow on that path new routing path is computed. After every transmission, residual energy $m$ of node $m$ changes, so after ' $\theta$ ' amount of transmission energy cost of each node is recalculated. With the updated energy costs the greedy heuristic path is recalculated and procedure is repeated until any node drain out its residual energy power.

\subsection{Energy Cost Function}

The objective is to find out best energy efficient algorithm that will lead to the maximization of system lifetime. The energy cost for a transmission from node $m$ to node $n$ is calculated by

$$
\mathrm{EC}_{(\mathrm{m}, \mathrm{n})=}\left(\mathrm{e}_{\mathrm{mn}}\right) \mathrm{m}^{-1}+\left(\mathrm{e}_{\mathrm{nm}}\right) \mathrm{n}^{-1}
$$

Where, $\mathrm{EC}_{(\mathrm{m}, \mathrm{n})}$ is the energy cost for transmitting a packet from node $\mathrm{m}$ to node $\mathrm{n}$. 


\subsection{Energy Cost Model}

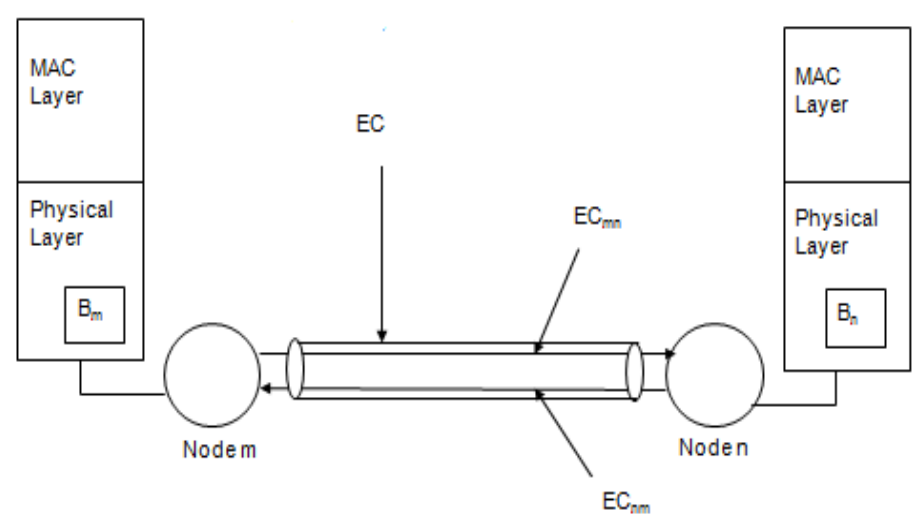

\subsection{Steps for creating energy efficient optimal path}

The routing path is computed variant of prim's MST algorithm [13]. The idea behind the algorithm is that every new node added to the greedy heuristic optimal path has the minimum cost to reach the base station. The algorithm works as follows:

Step (1): Initially we assume that base station is in optimal path. Base station can add any node if energy cost for a transmission from base station to one of its neighbor node is minimum, and suppose this neighbor node is i then create a link between base station and node $i$. Then node $i$ is also included in optimal path.

Step (2): The next link (i, j) to be added is such that $i$ is a node already included in a optimal path, $j$ is a node not yet included, and the energy cost of $(i, j)$ is minimum among all links ( $\mathrm{p}, \mathrm{q})$ such that node $\mathrm{p}$ is in the optimal path and node $\mathrm{q}$ is not in the optimal path.

Step (3): If any link (i, l) has minimum energy cost and energy cost of this link is also minimum among all links (p, q) where node $\mathrm{p}$ is in the optimal path and node $\mathrm{q}$ is not in optimal path then link (i, 1$)$ is added in path but after adding this link if create a cycle in optimal path then this link is not included in a path (fig. 1(a)).

Step (4): Select another link (BS, k) where BS is a node already included in a optimal path, and $\mathrm{k}$ is a node not yet included and energy cost is greater than link (i, l) but minimum among all links (p, q) such that node $\mathrm{p}$ is in path and node $\mathrm{q}$ is not in path.

Step (5): Repeat this procedure until all nodes of the graph have been added to the optimal path, a greedy heuristic path is constructed for the network.

Step (6): After transmitting $\theta$ amount of data in greedy heuristic path, the new optimal path is computed. Because after transmitting the data, residual energy of all the nodes are decreases and energy cost increase.

Step (7): Suppose in network 1(b) all the nodes can transmits the data packets and let energy cost of all the links will increases by 0.5 . So after $\theta$ amount of transmission minimum energy cost path will be recalculated shown in fig. 2(b). All the traffic flow should follow this new minimum energy cost path. 
Step (8): Repeat these steps until the first node in the network dies.
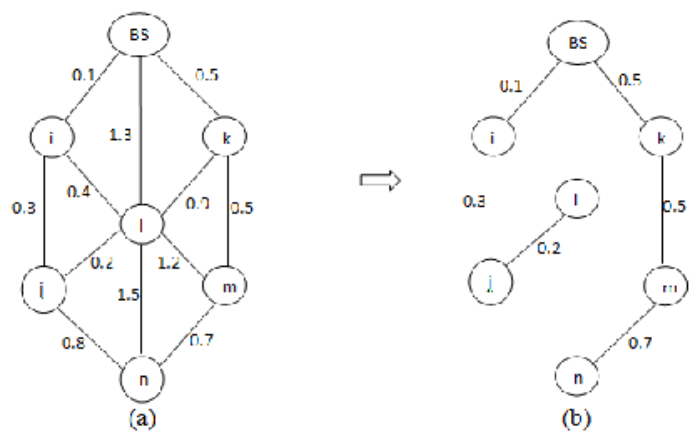

(1)

Figure 1. Minimum energy cost path

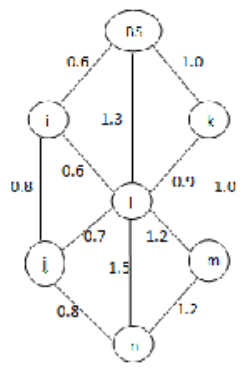

(a)

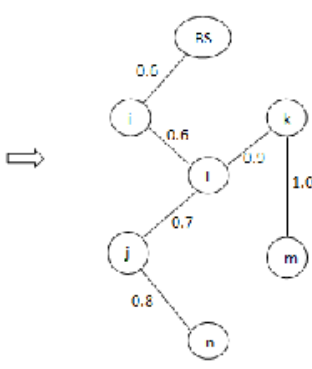

(b)

(2)

Figure 2. Minimum energy cost path after $\theta$ amount of transmission

\section{Performance Evaluations}

We have carried out extensive simulation studies of the proposed algorithm to evaluate its performance, and compared its performance with Ad-hoc on Demand Distance Vector routing algorithm (AODV). The AODV protocol is one of the reactive routing protocols that can construct the route when data transmission is required. In this protocol, a source node broadcasts the route request (RREQ) packet to the entire network, and all the nodes rebroadcast the received RREQ packet immediately. Therefore, we use the AODV protocol as the basic protocol since its operation is quite simple. In order to observe the affect of static parameter on AODV we have consider the platform's based simulation on Qualnet version 5.0 which is a standard tool set used sensor networks standards.

\subsection{Simulation Model}

In our simulation, we have varied the number of nodes from 10 to 50, which are randomly deployed using uniform distribution in different parts of deployment area with a fixed density. Assume that transmission range of each node is limited by 10 meter. The packet size was kept at 80 Bytes. We used Constant Bit Rate (CBR) as traffic source with average packet rate 0.5 packets /sec. Each node has initial energy $E_{\mathrm{i}}=100 \mathrm{~J}$. By experiment, we find that the suitable value of $\theta$ to be 10 packet and we have set the duration of each round to 1000 seconds. The input data is generated randomly in every second duration at each node. 
Table 1 Configuration Table

\begin{tabular}{|l|l|}
\hline Simulation Time & 100 Seconds \\
\hline Terrain Area & 500 x 500 (meter) $^{2}$ \\
\hline Number of Nodes (Sensors) & 10 to 50 \\
\hline Remote Site (Access Point) & 1 (Base Station) \\
\hline Transmission Range & 10 meter \\
\hline Bandwidth & $2 \mathrm{Mbps}$ \\
\hline Channel Frequency & $2.4 \mathrm{GHz}$ \\
\hline Traffic Type & UDP \\
\hline Mobility & NONE \\
\hline Application Type & CBR \\
\hline
\end{tabular}

\subsection{Simulation Result}

In this section the performance of proposed EEMLR algorithm is analyze and compares it with an underlying ad hoc routing protocol. Due to its popularity and reactive property, we choose Adhoc On Demand Distance Vector (AODV) routing algorithm as the underlying protocol for our simulations. AODV is a source-initiated reactive (on-demand) protocol, which initiates a route discovery whenever a node requires a path to a destination

\subsubsection{Remaining Node Energy}

The remaining node energy of all sensors (10 nodes scenario) at the end of simulation has been plotted in figure 3. The graph shows that EEMLR has distributed overall energy over the entire network in a more balanced way. In EEMLR algorithm after $\theta$ amount of transmission new routing path is constructed so this algorithm balanced the energy for entire network. Therefore, EEMLR routing algorithm should consider not only energy efficiency, but also the amount of energy available in each sensor. For example, EEMLR uses different path after every $\theta$ amount of transmission. Excessively energy consumption of one path (node 2 and node 4 ) has been shared by another path (node 9 and node 10). From the results, the remaining battery capacity of nodes in AODV decreases very early. This is because the sensor nodes near the sink nodes consume a large amount of battery power to forward data packets from a sensor node which is located far from the sink node. Therefore, the sensor nodes far from the sink nodes cannot find the route to the sink node. If the route is not found, each sensor node tries to find it again. As results, many sensor nodes consume a large amount of battery power to find the route to the sink nodes.

\subsubsection{Energy consumption}

The plot for energy consumption vs. number of nodes of two routing algorithms is shown in Figure 4 . The total energy consumption includes energy consumption in transmission, reception, idle and sleep modes of operation. In our simulation energy consumption at idle mode is ignored 
International Journal Of Advanced Smart Sensor Network Systems ( IJASSN ), Vol 2, No.1, January 2012

and it is noticed that transmission consumes greater energy than reception for transferring data packets while calculating total energy consumption in our simulation. During sleep time, there is no energy consumption. The total energy consumption of two routing algorithms increases when number of nodes or traffic load increases. However, EEMLR algorithm performs better than AODV at all specified number of nodes variation due to its low routing overhead as shown in Figure 4.7.

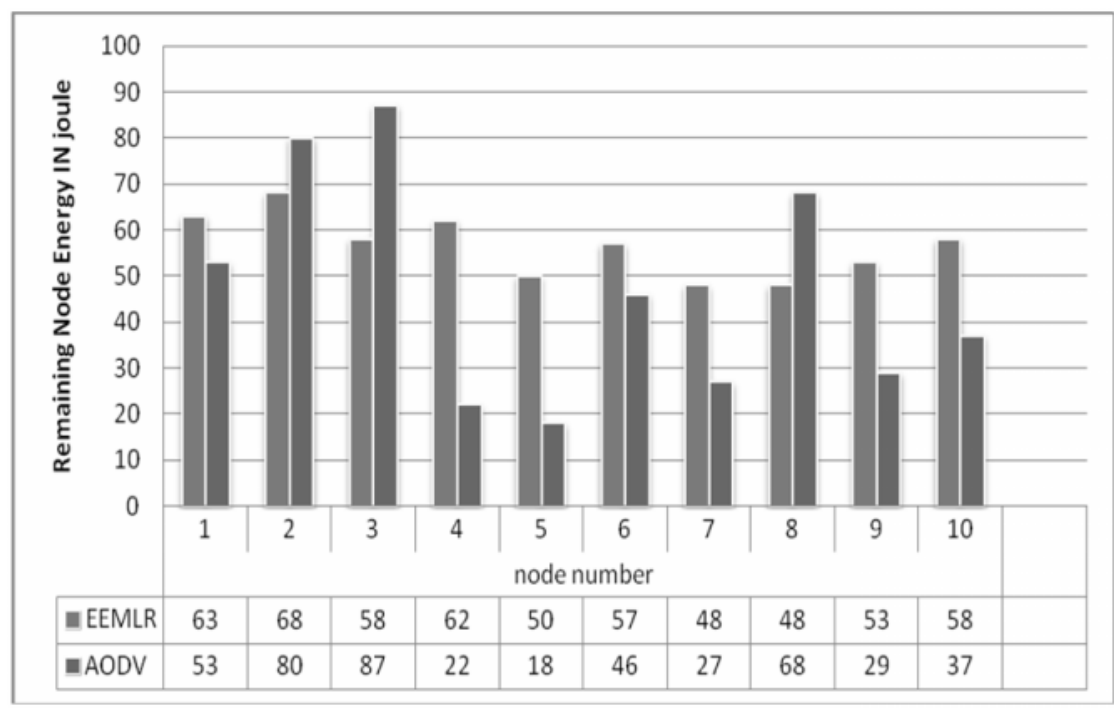

Figure 3. Remaining energy of nodes after simulation

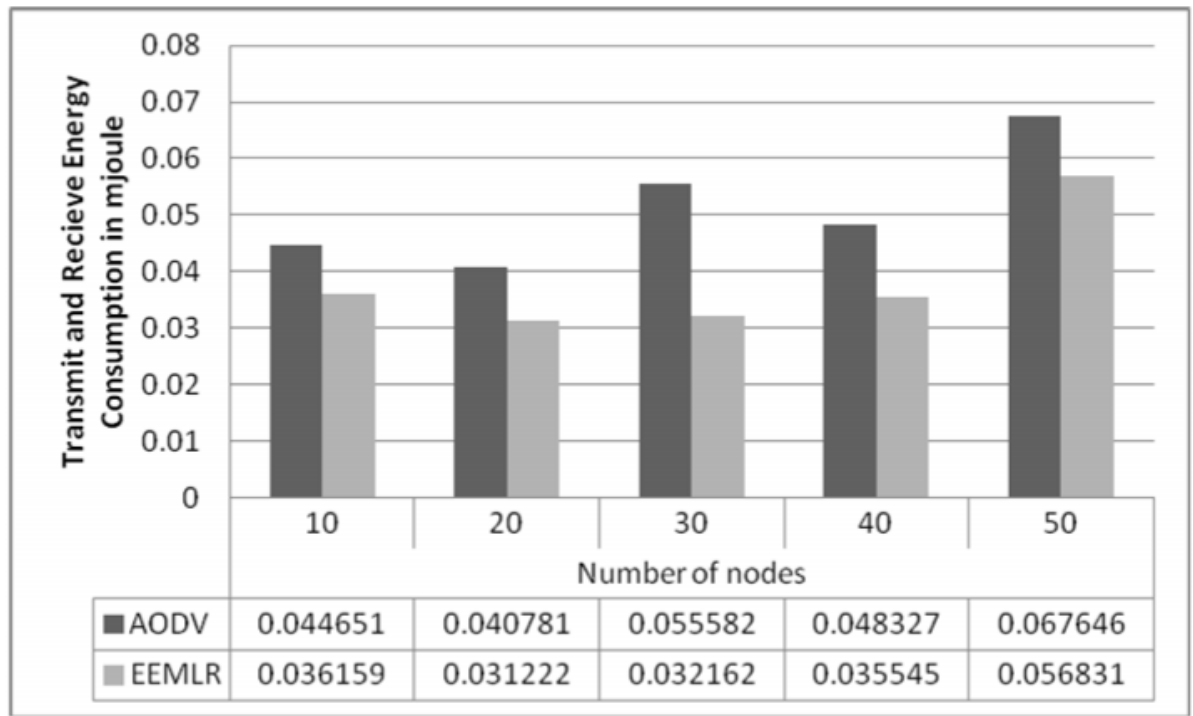

Figure 4. Energy consumption vs No. of node

\subsubsection{Packet Delivery ratio}

Figure 5 gives percentage packets delivered in each round using EEMLR and AODV approach for WSNs. It is to be noted that EEMLR algorithm consistently gives higher percentage of packets delivered in comparison to AODV algorithm. As shown in figure 5, EEMLR outperforms the AODV because of limited congestion due to less routing overhead. It is to be noted that 
International Journal Of Advanced Smart Sensor Network Systems ( IJASSN ), Vol 2, No.1, January 2012

percentage of packets delivered in EEMLR routing is slightly more than that in AODV routing. The ratio of data packets delivered to the destination and the data packets generated by the CBR sources are taken packet delivery ratio in our study.

\subsubsection{Average End to End Delay}

The plot for average end-to- end delay for varying number of nodes is shown in Figure 6. In AODV algorithm has higher end to end delay as compared to EEMLR algorithm because besides the actual delivery of data packets, the delay time is also affected by route discovery, which is the first step to begin a communication session in AODV. The average end- to- end delay of a packet depends on route discovery latency, besides delays at each hop and the number of hops. At few nodes, queuing and channel access delays do not contribute much to the overall delay. The average end to end delay is lower when total number of nodes in simulation scenario is 10 for both routing algorithms but it increases with increasing number of nodes. In AODV algorithm end to end delay increases very rapidly when number of nodes and data traffic increases.

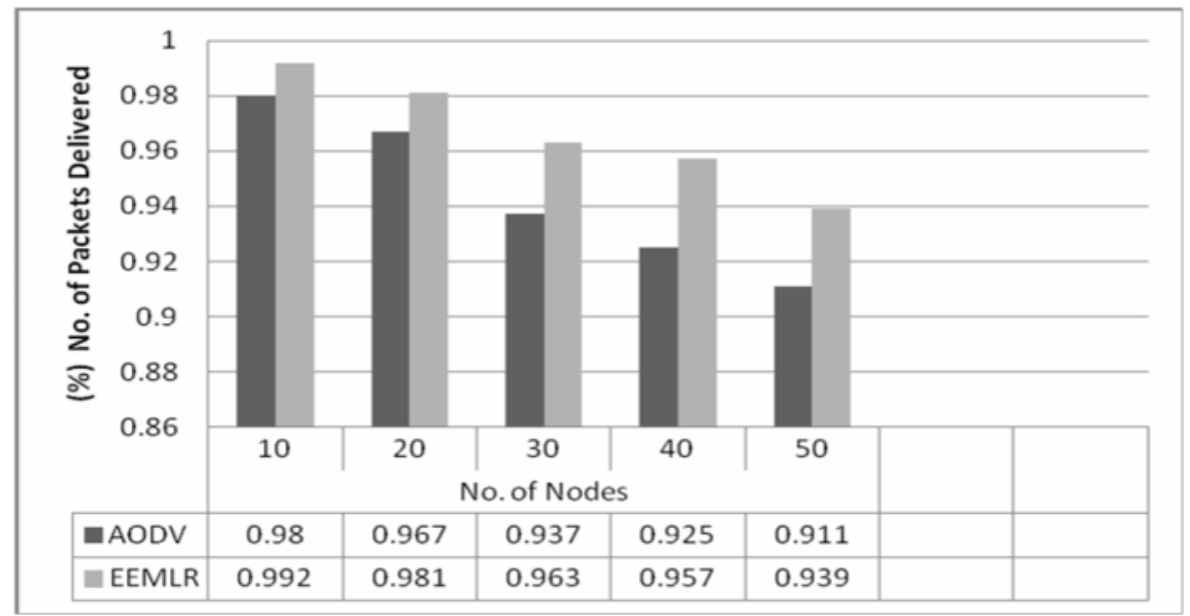

Figure 5. Packet delivery ratio for varying number of nodes

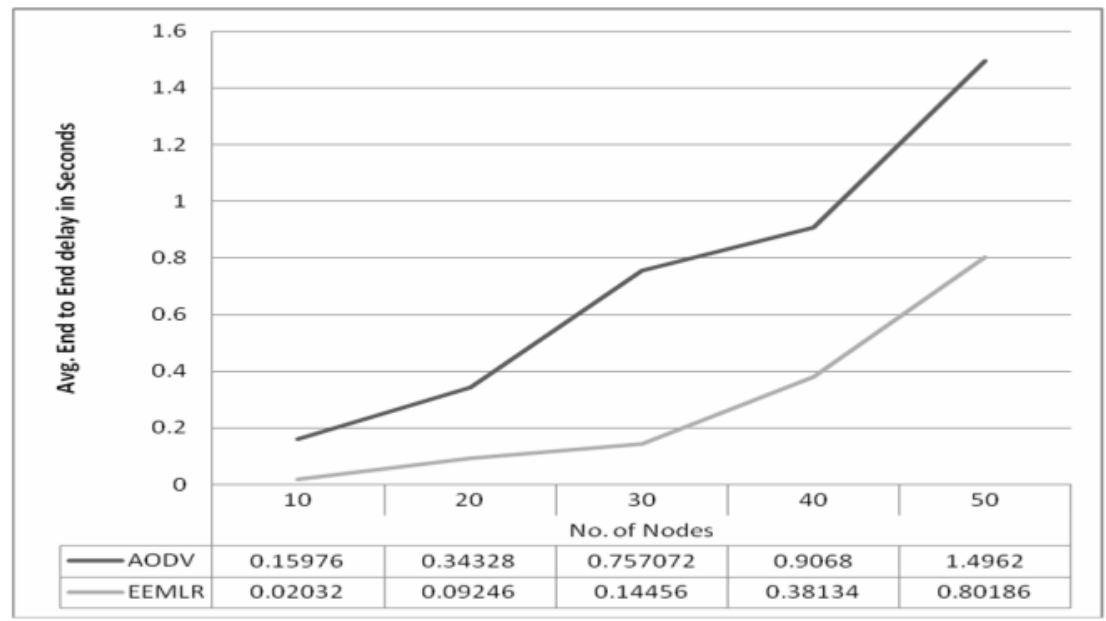

Figure 6. Avg. end to end delay vs No. of nodes 


\section{Conclusions}

The energy supplies of nodes in wireless sensor network are not replaced and therefore nodes only participate in the network for as long as they have energy, for that reason battery energy is the most important resource, so route the traffic through the minimum energy path to the destination is fatal for the network because all the nodes in that path will drain out their battery power rapidly. Therefore it's not a feasible solution and instead of this solution forwards the traffic such that energy consumption is balanced among the nodes. Most of the energy aware routing algorithm only concerned energy efficiency of the nodes but proposed EEMLR present the heuristic measure, called energy cost, to balance the energy consumption rates among the nodes in proportion to their energy reserved.

The performance of EEMLR algorithm is compared with AODV algorithm. The performance of these protocols is compared on the basis of end-to-end delay, packet delivery ratio, routing overhead, throughput and remaining node energy.

From the simulation results, we have evaluated the performance of our protocol for different number of nodes and conclude that AODV algorithm has higher end to end delay as compared to EEMLR algorithm. We also conclude that a routing protocol with more routing overhead would consume more energy than the routing protocol with less routing overhead means AODV routing algorithm has higher energy consumption than EEMLR algorithm because of higher routing overhead. Finally we can conclude that data packet delivery in EEMLR routing is more than that using AODV routing, and energy consumption of nodes is also balanced in EEMLR algorithm.

\section{REFERENCES}

[1] V. Raghunathan, C. Schurghers \& M. Srivastava, (2002) "Energy-aware Wireless Microsensor Networks", IEEE Communication Magazine, pp. 40-50.

[2] G. Pottie, W. Kaiser, (2000) "Wireless Integrated Network Sensors, Communication of ACM", Vol. 43, no. 5, pp. 51-58.

[3] Singh, Woo \& Raghavendra, (1998) Power-aware routing in mobile ad hoc networks, 4th Annual IEEE/ACM Int. Conf. Mobile Computing and Networking, pp. 181-190, Dallas, TX .

[4] Chang \& Tassiulas, (1999) Routing for maximum system lifetime in wireless ad hoc networks, 37th Annual Allerton Conf. Communication, Control, and Computing, Monticello, IL.

[5] Chang \& Tassiulas, (2000) Energy conserving routing in wireless ad hoc networks, in Proc. IEEE INFOCOM, pp. 22-31, Tel Aviv, Israel.

[6] Li, Aslam \& Rus, (2001) Online power-aware routing in wireless ad hoc networks, IEEE/ACM Int. Conf. Mobile Computing and Networking (MobiCom 2001), Rome, Italy.

[7] Chang \& Tassiulas, (2004) Maximum Lifetime Routing in Wireless Sensor Networks, IEEE/ACM Transactions on Networking", Vol. 12, issue 4.

[8] Ergen \& Varaiya, (2007) Energy Efficient Routing with Delay Guarantee for Sensor Networks, Wireless Networks, Vol. 13, No. 5, P.P. 679-690.

[9] Pandana \& Ray Liu, (2005) "Maximum Connectivity and Maximum Lifetime Energy-aware Routing for Wireless Sensor Network," IEEE GLOBOCOM.

[10] kar, Kodialam, Lakshman \& Tassiulas, (2003) Routing for Network Capacity Maximization in Energy-constained Network, IEEE INFOCOM.

[11] Anastasi, Conti, Francesco, \& Passarella, (2009) Energy conservation in wireless sensor networks: A survey, Ad Hoc Networks, vol. 7, No. 3, pp. 537-568.

[12] Ok, Lee, Mitra \& Kumara, (2009) Distributed Energy Balanced Routing for Wireless Sensor Networks, Computer \& Industrial Engineering, vol. 57, issue 1, P.P. 125-135.

[13] Horowitz, Sahni \& Rajasekharan,(2008) Fundamentals of Computer Algorithms, Second Edition, Silicon Press (2008) 
International Journal Of Advanced Smart Sensor Network Systems ( IJASSN ), Vol 2, No.1, January 2012

\section{Authors}

Mr. Sourabh Jain is Assistant professor in Gyan Ganga Institute of Technology and Science (GGITS) Jabalpur, India. He has completed his M. Tech. degree From MANIT, Bhopal, India. His general research interests include WSNs, Ad hoc Network and Information Security.

Mr. Praveen Kaushik is Assistant Professor in Maulana Azad National Institute of Technology (MANIT) Bhopal, India. He is pursing PhD in WSN. His general research interests include WSNs, Ad hoc Network and Wireless Communication.

Dr. Jyoti Singhai is Associate professor in Maulana Azad National Institute of Technology (MANIT), Bhopal, India. She holds Ph..D degree from MANIT, India. Her general research interests include wireless communication, image processing, WSN and network security.
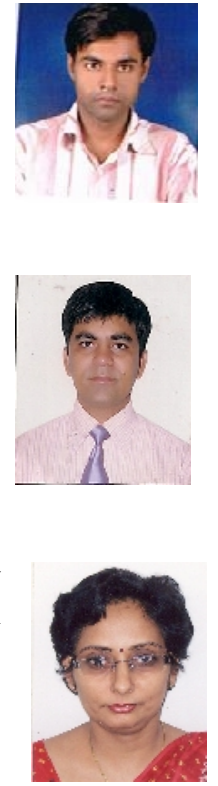